\title{
SYMMETRIC RIEMANN SURFACES, TORSION SUBGROUPS AND SCHOTTKY COVERINGS
}

\author{
BLAISE HELTAI
}

\begin{abstract}
We consider a torsion-free Fuchsian group $G$ acting on $H$ which admits an orientation reversing involution $j$. That is, $j G j=G$. Let $T$ be the orientation preserving half of the torsion subgroup of the extended group $\langle G, j\rangle$.

By considering invariant homology basis elements of the surface $H / G$, we show that the surface $H / T$ is planar, and that the group $G / T$ acts on $H / T$ as a Schottky group.
\end{abstract}

A closed Riemann surface $S$ is said to be symmetric if it admits an anticonformal involution $J$. If $J$ has fixed points on $S$, we say that it is a reflection on $S$.

The study of symmetric surfaces was initiated by Klein $[\mathbf{K}]$ who observed that a symmetric Riemann surface is the complex locus of a real algebraic curve. $S$ admits a reflection if the curve has a nonempty real locus.

The reflection on $S$ may be lifted to a hyperbolic reflection $j$ on the hyperbolic plane $H$. The uniformizing Fuchsian group $G$ for $S$ is conjugated to itself by $j$. We say that $G$ is a symmetric Fuchsian group. Such groups have recently been studied in various contexts by Singerman, Alling and Greenleaf, and Sibner. In a different vein, Sibner has proved that a symmetric surface has a symmetric Schottky uniformization [S2]. This will be proved in the present paper as well, using entirely different techniques. More to the point, we attempt to unify several ideas by showing that a symmetric Fuchsian uniformization gives rise naturally to a symmetric Schottky uniformization.

Since the reflection $j$ normalizes the group $G$, the extended group $\hat{G}=\langle G, j\rangle$ acts discontinuously on the universal cover $H$ of $S$. We define $\hat{T}$ to be the torsion subgroup of $\hat{G}$ and define $T$ to be the orientation preserving half of $T$. We say that $T$ is the harmonic subgroup of $G$ associated to $j$. We will show that $H / T$ (and of course $H / \hat{T}$ ) is a planar surface, and using a characterization of Schottky coverings developed in $\S 2$, we will prove:

THEOREM. G/T acts as a Schottky group on $H / T$.

This paper includes part of the author's dissertation $[\mathbf{H}]$ written under the aegis of Bernard Maskit. His guidance was invaluable. The terminology "harmonic subgroup" was suggested by the referee.

Received by the editors February 7, 1986 and, in revised form, May 19, 1986.

1980 Mathematics Subject Classification (1985 Revision). Primary 30F35; Secondary 30F10. 
1. The classical fact concerning symmetric surfaces is

(1.1) HARnACK's TheOREM. IF $J$ is an anticonformal involution on the closed Riemann surface $S$ of genus $g$, then the fixed point set of $J$ is either empty or consists of $s+1$ disjoint simple curves $c_{0}, \ldots, c_{s}$ where $0 \leqslant s \leqslant g$.

Equally well known is the following extension to Harnack's Theorem. A proof may be found in [K-M].

(1.2) In addition to the curves $c_{0}, \ldots, c_{s}$ fixed by $J$ (if any), there exist simple closed curves $c_{s+1}, \ldots, c_{s+t}$ such that

(i) $\left\{c_{i}\right\}_{i=0}^{s+t}$ is a disjoint collection of curves,

(ii) $J\left(c_{i}\right)=c_{i}$ for all $i$,

(iii) $S-\left\{c_{i}\right\}$ consists of two components, and

(iv) $J$ interchanges these components.

Notation. We write the triple $(g, s, t)$ to denote the symmetry type of a surface $S$ of genus $g$ with a fixed reflection $J$ which fixes (pointwise) $s+1$ curves $c_{0}, \ldots, c_{s}$ and rotates $t$ curves $c_{s+1}, \ldots, c_{s+t}$ as in (1.2).

2. We take a detour from symmetric surfaces to recall the definition of a Schottky group.

Let $\gamma_{i}, \gamma_{i}^{\prime}(i=1, \ldots, p)$ be disjoint Jordan curves in $\hat{\mathbf{C}}$ which all bound a domain $\Delta$. Let Ext $\gamma$ denote the component of $\hat{\mathbf{C}}-\gamma$ which contains $\Delta$ and let Int $\gamma$ be the other component. If there exist Möbius transformations $g_{i}$ with the property $g_{i}\left(\right.$ Int $\left.\gamma_{i}\right)=\operatorname{Ext} \gamma_{i}^{\prime}$, then the group $\Gamma$ generated by $\left\{g_{i}\right\}$ is a free group acting discontinuously on an open dense subset $\Omega(\Gamma)$ of $\hat{\mathbf{C}}$ and $\Gamma$ is a Schottky group. The orbit space of this action on $\Omega$ is a closed surface of genus $p$ and $\Delta$ is a fundamental domain for it.

The classical retrosection theorem asserts that for any closed Riemann surface $X$, there exists a Schottky group $\Gamma$ whose orbit space $\Omega / \Gamma$ is conformally equivalent to $X$. Our immediate aim here is to give a topological characterization of such Schottky coverings $\Omega \rightarrow X$. In order to do so we must enlarge our point of view slightly.

A surface $Z$ is planar if any simple closed curve in $Z$ divides $Z$. Equivalently, there exists a homology basis for $Z$ with all zero intersection numbers.

If $X$ is a closed surface, $Z$ a planar surface, and $Z \rightarrow X$ an unbranched regular covering with covering group $\Gamma$, then we say that $Z \rightarrow X$ is a Schottky-like covering if there exists an embedding $\Psi: Z \rightarrow \hat{\mathrm{C}}$ such that $\Psi \Gamma \Psi^{-1}$ is a Schottky group.

The essential fact about planar coverings is contained in the following. We state only a special case.

Planarity Theorem (MASkit [M1]). Given a closed surface $X$ and an unbranched regular covering $p: Z \rightarrow X$ where $Z$ is planar, there exist disjoint, homotopically independent simple closed curves $L_{1}, \ldots, L_{m}$ whose representatives in $\pi_{1}(X)$ normally generate $p_{*} \pi_{1}(Z) \simeq(Z)$.

In other words, if we fix a base point $x_{0}$ in $X$ and take $l_{i}$ to be elements of $\pi_{1}\left(X, x_{0}\right)$ freely homotopic to $L_{i}$, the normal closure of $\left\langle l_{1}, \ldots, l_{m}\right\rangle$ is $p_{*} \pi_{1}\left(Z, z_{0}\right)$ 
where $p\left(z_{0}\right)=x_{0}$. We say that $\left\{l_{1}, \ldots, l_{m}\right\}$ normally generate $\pi_{1}\left(Z, z_{0}\right)$ in $\pi_{1}\left(X, x_{0}\right)$ and that $L_{1}, \ldots, L_{m}$ define the covering.

The implication of the Planarity Theorem to Schottky coverings is as follows. The techniques in (2.1) are largely due to Maskit, although the theorem has perhaps not been stated in this form. We thus include a proof.

(2.1) THEOREM. If $X$ is a closed surface of genus $g$, then $p: Z \rightarrow X$ is a Schottky-like covering with covering group $\Gamma$ if and only if $Z$ is planar and there exist $g$ disjoint, homologically independent simple closed curves $A_{1}, \ldots, A_{g}$ in $X$ which lift to simple closed curves in $Z$.

Proof. If $Z \rightarrow X$ is a Schottky covering, take $A_{1}, \ldots, A_{g}$ to be the images in $X$ of the defining Jordan curves $\gamma_{1}, \ldots, \gamma_{g}$ for the Schottky group.

Conversely, we view the $A_{i}$ as cycles in $H_{1}(X, Z)$ and form a homology basis $\left\{A_{1}, \ldots, A_{g}, B_{1}, \ldots, B_{g}\right\}$. Let $X^{*}$ be the surface obtained by cutting $X$ along the $A_{i}$. Then $X^{*}$ is connected, has genus 0 and $2 g$ boundary loops. Fixing a base point 0 in $X^{*}$, let $a_{i} \in \pi_{1}(X, 0)$ represent $A_{i}$. Denote the normal closure of $\left\{a_{1}, \ldots, a_{g}\right\}$ in $\pi_{1}(X)$ by $N . N$ is then the defining subgroup of some regular planar covering $Z_{N}$ of $Z$.

Since every loop $A_{i}$ lifts to a loop in $Z, Z_{N}$ covers $Z$. We will show that in fact $Z_{N}=Z$.

Let $W$ be any loop on $X$ disjoint from each $A_{i}$. Then $W$ divides $X^{*}$. Consider a component $K$ of $X^{*}-W . K$ has genus 0 and is bounded by $W$ and some subset of $\left\{A_{i}\right\}$ (with some $A_{i}$ 's possibly appearing twice).

We may take the base point 0 to be in $K$ and thus the embedding $K \subset X$ induces a homomorphism $\varphi: \pi_{1}(K, 0) \rightarrow \pi_{1}(X, 0)$.

Let $\left\{w, \alpha_{1}, \ldots, \alpha_{m}\right\}$ be a set of generators for $\pi_{1}(K, 0)$ where $w$ represents $W$ and $\alpha_{i}$ is homotopic to a boundary curve of $K$. Since $K$ is an $m+1$ holed sphere, $w^{-1}=\alpha_{1} \alpha_{2} \cdots \alpha_{m}$ and $\varphi\left(w^{-1}\right)=\varphi\left(\alpha_{1} \alpha_{2} \cdots \alpha_{m}\right)$. But $\varphi\left(\alpha_{i}\right)$ represents a boundary curve of $K$ and hence $\varphi\left(\alpha_{i}\right)$ is conjugate to $a_{j}$ for some $j$. Thus $\varphi\left(w^{-1}\right)$, which represents $W$ in $\pi_{1}(X)$, is an element of $N$, the normal closure of $\left\{a_{j}\right\}$. We conclude that $W$ lifts to a loop in $Z_{N}$. By the Planarity Theorem, we have $Z_{N}=Z$.

We now follow [M2]. Let $x_{0}$ be a point in $\operatorname{Int}\left(X^{*}\right)$ and $z_{0} \in P^{-1}\left(x_{0}\right) \subset Z$. If $x \in X^{*}$, let $\rho_{1}$ be a path in $X^{*}$ from $x_{0}$ to $x$. Take the lift $\tilde{\rho}_{1}$ which has one endpoint at $z_{0}$. If $\rho_{2}$ is another path in $X^{*}$ from $x_{0}$ to $x$, then $\rho_{2}^{-1} \rho_{1}$ is a loop in $X^{*}$. We have shown that any loop in $X^{*}$ lifts to a loop in $Z$. Hence $p^{-1} \mid X^{*}$ is a well-defined map.

Let $\Delta=p^{-1}$ (int $\left.X^{* \prime}\right)$. Then $p \mid \Delta$ is one-to-one. If $\tilde{A}_{i}$ is a lift of $A_{i}$ which bounds $\Delta$, by lifting $B_{i}$ into $\Delta$ we see that there exists another lift $\tilde{A}_{i}^{\prime}$ of $A_{i}$ which also bounds $\Delta$. For each $i$, let $h_{i}$ be the element of the covering group $\Gamma$ which identifies $\tilde{A}_{i}$ and $\tilde{A}_{i}^{\prime}$. The lift of $B_{i}$ in $\Delta$ has its endpoints identified by $h_{i}$. Since the corresponding element $\left\{a_{1}, \ldots, a_{g}, b_{1}, \ldots, b_{g}\right\}$ generates $\pi_{1}(S, 0),\left\{h_{1}, \ldots, h_{g}\right\}$ generates $\Gamma$. Since $p \mid \Delta$ is one-to-one, $h_{i}(\Delta) \cap \Delta=\varnothing$. Thus $\Gamma$ "looks like" a Schottky group. 
In order to complete the proof, we recall the following.

(2.2) THEOREM (MASKIT [M3]). Let $\mathscr{D}$ be a plane domain and let $M$ be a group of conformal homeomorphisms of $\mathscr{D}$ onto itself. Then there exists a univalent function $\psi$, mapping $\mathscr{D}$ onto $\mathscr{D}^{\prime}$, so that every element of $\psi M \psi^{-1}$ is a Möbius transformation.

We may thus find a conformal map $\psi$ from $Z$ into $\hat{\mathbf{C}}$ such that $\psi h_{i} \psi^{-1}$ are Möbius transformations. $\psi \Gamma \psi^{-1}$ is then a Schottky group and hence $p: Z \rightarrow X$ is a Schottky-like covering.

3. We consider a Riemann surface $S$ of genus $g>1$ with a reflection $J$ and of symmetry type $(g, s, t)$. Let $H$ be the upper half-plane $\{z \in \mathbf{C} \mid \operatorname{Im} z>0\}$. Then $S=H / G$ where $G$ is a freely acting group of Möbius transformations on $H$. That is, $G$ is a torsion free Fuchsian group. We impose the Poincaré metric on $H$, so that $H$ becomes a model for the hyperbolic plane and $S$ a surface of curvature -1 . We call the geodesics in $\mathrm{H}, \mathrm{H}$-lines.

Since $J$ is an isometry of $S$ with this metric, the curves $c_{0}, \ldots, c_{s}$ given in Harnack's Theorem are geodesic. Let $j$ be a lift of $J$ to an orientation reversing isometry of $H$. Let $\hat{G}=\langle G, j\rangle$, the group generated by $G$ and $j$, and observe that $S / J \simeq H / \hat{G}$. Moreover $S$ is a two-fold covering of $S / J$ and hence $[\hat{G}: G]=2$.

Let $x$ be a fixed point of $J$ on $S, z \in H$ lying over $x$. Then either $j$ fixes $z$ (in which case $j$ is an $H$-reflection) or $j(z)=z^{\prime}$. Since $z^{\prime}$ also lies over $x$, there exists $g \in G$ with $g\left(z^{\prime}\right)=z$. Note that $g j$ fixed $z$, hence so does $(g j)^{2}$. But since $[\hat{G}: G]=2,(g j)^{2} \in G$. But $G$ contains no elliptic elements ( $G$ acts freely on $H$ ), hence $g j$ is an involution. As $g j$ fixes a point in $H$, it must be a reflection through an $H$-line. Replacing $g j$ by $j$ if necessary, we summarize:

LemMa. The reflection $J$ on $S$ can be lifted to a hyperbolic reflection $j$ on $H$. $\hat{G}=\langle G, j\rangle$ is a $Z_{2}$-extension of $G$ and $H / \hat{G} \simeq S / J$.

Set $\hat{T}=$ torsion subgroup of $\hat{G}$. That is, $\hat{T}$ is generated by all elements of finite order in $\hat{G}$. Since $G$ is torsion free and $[\hat{G}: G]=2, \hat{T}$ is in fact generated by all reflections in $\hat{G}$. $\hat{T}$ is normal in $\hat{G}$.

We set $T=$ orientation preserving half of $\hat{G}$. As $G$ is the orientation preserving half of $\hat{G}, T$ is a normal subgroup of $G$. We may now precisely state the main theorem of this paper.

(3.1) THEOREM. $H / T \rightarrow S$ is a Schottky-like covering with covering group $G / T$.

We remark that for genus $=1, H$ replaced by $\mathbf{C}, G$ a Euclidean lattice group, and $T$ defined analogously, Theorem (3.1) is still true. The proof is elementary.

4. We will first establish that $H / T$ is a planar surface.

Let $R$ be the set of reflections in $\hat{G}$. For $r \in R$, let $A(r)$ be its reflection axis. $A(r)$ is an $H$-line. For $r \neq r^{\prime}$ in $R, A(r) \cap A\left(r^{\prime}\right)=\varnothing$ since $G$ contains no elliptic elements. The set $\{A(r): r \in R\}$ divides $H$ into connected components. Choose one which has $A(j)$, the axis of $j$, lying on its boundary: call this domain $\hat{D}$. Double $\hat{D}$ using the reflection $j$, and call the resulting domain $D$. Note that $D$ is bounded by reflection axes and that $A(j)$ splits $D$ in "half." 
(4.1) LEMMA. $D$ is a fundamental domain for $T$.

Proof (SKeTCH). $\hat{D}$ is necessarily an infinite sided polygon (with no nonideal vertices). Let $\left\{A(j), A\left(r_{1}\right), A\left(r_{2}\right), \ldots\right\}$ be its sides. Then $R_{0}=\left\{j, r_{1}, r_{2}, \ldots\right\}$ in fact generates $\hat{T}$ and, appealing to Poincaré's Polygon Theorem, $\hat{D}$ is a fundamental domain for $\left\langle R_{0}\right\rangle=\hat{T}$.

If $\tau \in T$, then $\tau$ is a word in $R_{0}$ with an even number of letters since $\tau$ preserves orientation. If $\tau=r_{1} r_{2}, r_{1} r_{2}=r_{1} j j r_{2}=\left(j r_{1}\right)^{-1} j r_{2}$, so $\tau$ is a word in $j R_{0}$. Proceed inductively to find that any $\tau$ in $T$ is a word in $j R_{0}$. Then, as above, $j R_{0}$ generates $T$, and $D$ is a fundamental domain for $\left\langle j R_{0}\right\rangle$, thus for $T$.

(4.2) LEMMA. $H / T$ is a planar surface.

Proof. Using the notation from (3.1) $D$ is bounded by reflection axes $\left\{A\left(r_{i}\right), j\left(A\left(r_{i}\right)\right)\right\}$. The transformations $j r_{i}$ generate $T$ and pair the sides $A\left(r_{i}\right)$ with $j\left(A\left(r_{i}\right)\right)$. For each $i, j r_{i}$ is either a hyperbolic transformation if $A\left(r_{i}\right), A(j)$ do not meet on $\partial H$, or $j r_{i}$ is a parabolic transformation otherwise. In the former case, let $\mathscr{A}_{i}$ be the $H$-line invariant under $j r_{i}$, and in the latter, let $\mathscr{A}_{i}$ be an invariant horocycle of $j r_{i}$ disjoint from all other $\mathscr{A}_{k}$.

$\left\{\mathscr{A}_{i}\right\}$ is a disjoint collection of curves in $H$ which project to disjoint simple closed curves in $H / T$. Moreover $\mathscr{A}_{i}$ is invariant under $j r_{i}$ and $\left\{j r_{i}\right\}$ generates $T$. Hence these projected loops on $H / T$ generate $H_{1}(H / T, Z)$. Since they are disjoint, $H / T$ is planar.

5.

(5.1) THEOREM. Let $S$ be a closed surface with a reflection $J$ of symmetry type $(g, s, t)$. Then there exist simple closed curves $A_{1}, \ldots, A_{g}$ on $S$ with the following properties:

(i) $J\left(A_{i}\right)=A_{i}, r=1, \ldots, g$,

(ii) the set $\left\{A_{1}, \ldots, A_{g}\right\}$ is homologically independent, and

(iii) each $A_{i}$ lifts to a simple closed curve in $H / T$.

The proof of Theorem (5.1) relies on constructing the $A_{i}$. We begin by fixing some notation from Harnack's Theorem and its extension (1.2). We have curves $c_{0}, \ldots, c_{s}$ fixed by $J$ and $c_{s+1}, \ldots, c_{s+t}$ rotated by $J$. These curves divide $S$ into two homeomorphic subsurfaces whose closures we call $\Sigma$ and $J \Sigma$. Each is a surface with $h=\frac{1}{2}(g-n+1)$ handles and $n$ boundary curves where $n=s+t+1$. The key ingredient in the construction of the $A_{i}$ may be summarized in the following technical lemma.

(5.2) LEMMA. There exist mutually disjoint paths on $\Sigma$ as follows:

(i) For each fixed curve $c_{i}(i=1, \ldots, s)$ on $\partial \Sigma$, there exists a simple path $u_{i}$ from $c_{0}$ to $c_{i}$.

(ii) For each rotated curve $c_{i}(i=s+1, \ldots, s+t)$, there exist two simple paths $v_{i}$, $w_{i}$ from $c_{0}$ to $c_{i}$ such that if $v_{i}$ meets $c_{i}$ at $z$ then $w_{i}$ meets $c_{i}$ at $J(z)$ (which is a half twist from $z$ along $c_{i}$ ). Moreover, $w_{i}$ is freely homotopic to $v_{i}$.

(iii) For each handle on $\Sigma$ there exist two homotopically distinct simple paths which begin and end on $c_{0}$. Call these paths $p_{i}, q_{i}(i=1, \ldots, h)$.

(iv) The collection $\left\{u_{i}, v_{i}, p_{i}, q_{i}\right\}$ does not disconnect $\Sigma$. 


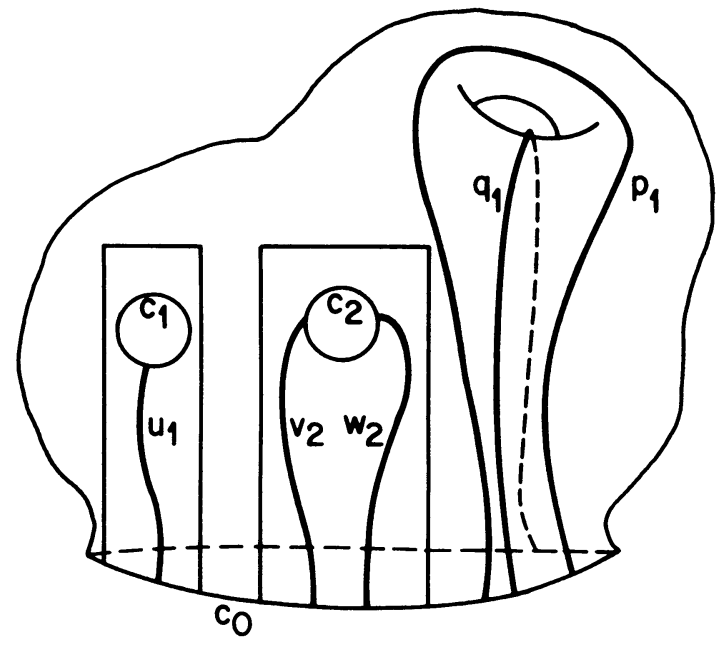

FIGURE 1

Proof. Cap off $\Sigma$ by attaching a disk $\Delta$ along $c_{0}$. Let $\hat{\Sigma}=\Sigma \cup_{c_{0}} \Delta$ and fix a point $*$ in $\Delta$. Choose a standard set of generating loops for $\pi_{1}(\hat{\Sigma}, *)$. That is, the set consists of $2 h+n-1$ simple closed curves, meeting only at $*$ where $\hat{p}_{i}, \hat{q}_{i}$ correspond to the handles on $\hat{\Sigma}, U_{i}$ are curves which go around the boundary curves $c_{i}(i=1, \ldots, s)$, and $V_{i}$ around the $c_{i}(i=s+1, \ldots, s+t)$. Let $\hat{u}_{i}$ be a path from * to $c_{i}(i=1, \ldots, s)$ which meets $U_{i}$ only at *.

For $i=s+1, \ldots, s+t$, let $\hat{v}_{i}$, $\hat{w}_{i}$ both be simple paths from $*$ to $c_{i}$ which meet $V_{i}$ and each other only at $*$ and which meet $c_{i}$ a half twist apart.

Now remove the disk $\Delta$ along $c_{0}$. What remains of the paths $\hat{u}_{i}, \hat{v}_{i}, \hat{w}_{i}, \hat{p}_{i}, \hat{q}_{i}$ we will call $u_{i}$ ( $F$-paths, for fixed curves); $v_{i}, w_{i}$ ( $R$-paths, for rotated curves); $p_{i}, q_{i}$ ( $H$-paths, for handles) (see Figure 1$)$.

To check property (ii), observe that $V_{i}$ divides $\hat{\Sigma}$ into two components and $\hat{v}_{i}, \hat{w}_{i}$ both lie in the one homeomorphic to a cylinder and are homotopic there.

We verify (iv) by observing that $\left\{\hat{p}_{i}, \hat{q}_{i}, U_{i}, V_{i}\right\}$ does not disconnect $\hat{\Sigma}, \hat{u}_{i} \simeq U_{i}$, $\hat{v}_{i} \simeq V_{i}$, and consequently removing $\left\{p_{i}, q_{i}, u_{i}, v_{i}\right\}$ from $\Sigma$ leaves a connected surface.

Note that adding the $w_{i}$ to this set will disconnect $\Sigma$ as $v_{i} \simeq w_{i}$.

We proceed with the proof of (5.1).

Double the paths $\left\{u_{i}, v_{i}, w_{i}, p_{i}, q_{i}\right\}$ from (4.2) into $J(\Sigma)$ via $J$. Then on $S=\Sigma \cup$ $J \Sigma$ we have a disjoint collection of simple paths (see Figure 2); after reindexing

$$
A_{i}= \begin{cases}u_{i} \cdot J\left(u_{i}\right), & i=1, \ldots, s, \text { called } F \text {-loops, } \\ v_{i} \cdot J\left(w_{i}\right) \cdot w_{i} \cdot J\left(v_{i}\right), & i=s+1, \ldots, s+t, \text { called } R \text {-loops, } \\ p_{i} \cdot J\left(p_{i}\right), & i=s+t+1, \ldots, s+t+h, \\ q_{i} \cdot J\left(q_{i}\right), & i=s+t+h+1, \ldots, s+t+2 h, \quad \text { called } H \text {-loops. }\end{cases}
$$




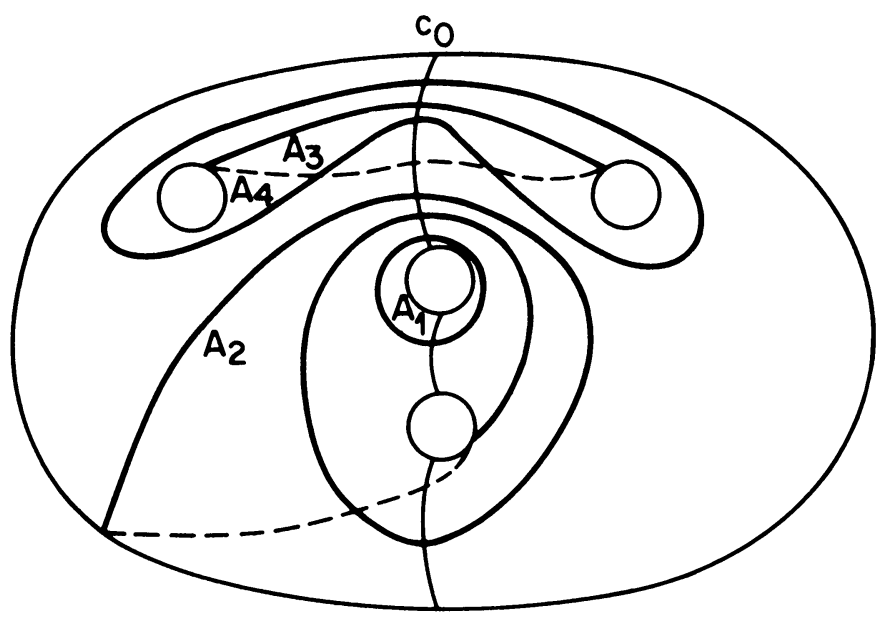

FIGURE 2

Note that $s+t+2 h=g$, that each $A_{i}$ is in fact closed, and $J\left(A_{i}\right)=A_{i}$ by construction. $\left\{A_{1}, \ldots, A_{g}\right\}$ is independent in homology if $S-\left\{A_{1}, \ldots, A_{g}\right\}$ is connected. From (5.2) it follows that the $F$-loops and $R$-loops together fail to disconnect $S$.

Recall also from (5.2) that each pair $v_{i}, w_{i}$ disconnects $\Sigma$, with one component homeomorphic to a disk. Let $x$ be a point in this disk. Similarly the pair $J\left(v_{i}\right), J\left(w_{i}\right)$ bounds a disk in $J \Sigma$. Notice that $J(x)$ cannot be in this disk as $J$ rotates the common boundary component of the two disks. But by taking a path from $x$ to this common boundary, it is easy to get a path from $x$ to $J(x)$ which meets no other loops $A_{i}$. Hence the entire collection $\left\{A_{1}, \ldots, A_{g}\right\}$ fails to disconnect $S$.

From $\$ 4$ recall the fundamental domain $D$ for $T$. Assume, with no loss of generality, that $A(j)$, the axis of $j$, is a lift of $c_{0} . D$ is bounded by lifts of all fixed curves $c_{0}, \ldots, c_{s}$.

Let $A_{i}$ be an $R$-loop or an $H$-loop on $S$. Lift $A_{i}$ to $\tilde{A_{i}}$ in $D$ and note that since $A_{i}$ meets no fixed curve but $c_{0}, \tilde{A}_{i}$ will meet $A(j)$ in the interior of $D$ and must meet $\partial D$ at $j$-equivalent points on some $A\left(r_{k}\right), j\left(A\left(r_{k}\right)\right)$ (both lifts of $\left.c_{0}\right)$.

Similarly, if $A_{i}$ is an $F$-loop, $\tilde{A}_{i}$ will meet $\partial D$ at $j$-equivalent points on some $A\left(r_{k}\right), j\left(A\left(r_{k}\right)\right)$ (both lifts of $\left.c_{i}\right)$. In either case, $A_{i}$ lifts to a loop in $\operatorname{cl} D /\langle j\rangle \simeq H / T$.

6. We conclude the proof of Theorem (3.1). From (4.2), $H / T$ is a planar surface and from parts (ii) and (iii) of (5.1), there exist homologically independent $\left\{A_{1}, \ldots, A_{g}\right\}$ which lift to loops in $H / T$. We apply (2.1) to conclude that the covering is Schottky-like with covering group $G / T$.

We may remark finally that part (i) of (4.2) implies that the "Schottky group" $G / T$ is symmetric. That is, the involution $j$ lifts to an involution $J$ on $H / T$ which commutes with the action of $G / T$. 


\section{REFERENCES}

[H] B. Heltai, Ph.D. Dissertation, State Univ. of New York at Stony Brook, 1984.

[K] F. Klein, On Riemann's theory of algebraic functions and their integrals, Dover, 1963.

[K-M] I. Kra and B. Maskit, Bases for quadratic differentials, Comment. Math. Helv. 57 (1982), $603-626$.

[M1] B. Maskit, A theorem on planar covering surfaces with applications to 3-manifolds, Ann. of Math. (2) 81 (1965), 341-355.

[M2] _ A characterization of Schottky groups, J. Analyse Math. 19 (1967), 227-230.

[M3] _ The conformal group of a plane domain, Amer. J. Math. 90 (1968), 718-722.

[S1] R. J. Sibner, Symmetric Fuchsian groups, Amer. J. Math. 90 (1968), 1237-1259.

[S2] _ Uniformizations of symmetric Riemann surfaces by Schottky groups, Trans. Amer. Math. Soc. 116 (1965), 79-85.

[SN] D. Singerman, Symmetries of Riemann surfaces with large automorphism group, Math. Ann. 210 (1974), 17-32.

Department of Mathematics, Indiana University, Bloomington, Indiana 47405

Current address: AT \& T Bell Laboratories, 600 Mountain Avenue, Murray Hill, New Jersey 07974 